\title{
Prelmplantation Factor promotes Oligodentrocyte differentiation by modulating NCOR2 and long non-coding RNA H19 of the Oligodendrocyte Progenitor Cells
}

\author{
Author: 1) Spinelli M., 2) Ornaghi S., 1) Schoeberlein A., 3) Keller I., 7) Barnea E., 2) Paidas M., \\ 1) Surbek $D ., 1,2,6)$ Mueller $M$. \\ Clinic: 1) Obstetrics and Gynecology and Biomedical Research, Inselspital, Bern University \\ Hospital, University of Bern, 2) Obstetrics, Gynecology, and Reproductive Sciences, \\ 3) BioMedical Research and Swiss Institute of Bioinformatics, University of Bern, \\ 4) Radiology and Biomedical Imaging, 5) Department of Biomedical Engineering, \\ 6) Neurosurgery, and Cellular \& Molecular Physiology, 7) Research, Biolncept LLC, New York/ \\ 2,4,5,6 Yale University School of Medicine, New Haven
}

Introduction: Premature infants face multiple challenges including periventricular leukomalacia (PVL) and successful therapies are lacking. Oligodendrocyte Progenitor Cells (OPCs) give rise to myelin producing cells during brain development. Activation of dormant OPCs at the epigenetic level represents an attractive strategy. Long non-coding RNA H19 is a potential candidate to regulate epigenetic cell differentiation since it inhibits S-adenosylmethionine-dependent methyltransferases that methylate DNA at regulatory sites. Since synthetic Prelmplantation factor (sPIF) protects against multiple neuronal disorders, we posit that sPIF activates OPCs by tuning gene methylation dynamics through $\mathrm{H} 19$.

Methods: Cell lines (OPCs MO13.13) were treated with SPIF (200nM; 48h), and downstream differentiation markers were evaluated by quantitative RT-PCR. H19 loss and gain of function studies and genome-wide methylation profiling were performed. Two-tailed Student's t-test and Mann-Whitney tests were used in analysis with level of significance set at $p<0.05$.

Results: Both sPIF and the overexpression of $\mathrm{H} 19$ enhanced oligodendrocyte differentiation. In MO13.13s, sPIF increased mRNA expression of immature (OLIG2) and mature (MBP) oligodendrocyte markers in H19-dependent manner. Genome-wide methylation profiling reveals that $\mathrm{H} 19$ overexpression significantly de - methylated intron regions of the gene locus of NCOR2, a key regulatory cellular factor, leading to an increased expression. NCOR2 enhanced oligodendrocyte differentiation and OPCs knockdown for NCOR2, subjected to sPIF, were not able to differentiate.

Conclusion: sPIF activates OPCs and boosters myelin production by modulating $\mathrm{H} 19$ and the de-methylation of NCOR2 of the OPCs. Our results uncover an unanticipated regulatory circuit of SPIF involving broad epigenetic alterations by a single IncRNA that may underlie gene methylation dynamics of development and diseases. Given the FDA Fast Track designation and safety data of sPIF in First in Human Clinical Trial (ClinicalTrials.gov Identifier: NCT02239562), clinical trials to prevent or treat PVL can be envisioned. 